\title{
STRONGLY ASYMPTOTICALLY HYPERBOLIC SPIN MANIFOLDS
}

\author{
XIAO ZHANG
}

\section{Introduction}

In this note, we shall generalize Min-Oo's theorem of scalar curvature rigidity of strongly asymptotically hyperbolic spin manifolds.

Inspired by Witten's proof of the positive energy theorem for asymptotically flat manifolds, Min-Oo introduced strongly asymptotically hyperbolic manifolds and proved that any strongly asymptotically hyperbolic spin manifold of dimension $n \geq 3$ with scalar curvature $R \geq-n(n-1)$ is isometric to hyperbolic space [3]. This rigid theorem was extended later to locally asymptotically hyperbolic spin manifolds by Andersson and Dahl [1], and to odd-dimensional complex hyperbolic manifold by Herzlich [2].

Recall the hyperbolic space $\left(H^{n}, g_{0}\right)$ is $R^{n}$ endowed with the metric

$$
g_{0}=d r^{2}+\sinh ^{2}(r) d \Omega_{n-1}^{2}
$$

in polar coordinates, which has constant sectional curvature $K_{0}=-1$ and scalar curvature $R_{0}=-n(n-1)$. For $r>0$, we denote by $H_{r}$ the complement of a closed ball of radius $r$ around the origin, i.e., $H_{r}=H^{n}-\bar{B}_{r}(0)$. A smooth Riemannian manifold $\left(M^{n}, g\right)$ is said to be strongly asymptotically hyperbolic (with one end) if there exists a compact subset $K \subset M$ and a diffeomorphism $f: M_{\infty} \equiv M-K \rightarrow H_{r}$ for some $r>0$, such that, on $M_{\infty}$, the metric $g$ can be written as

$$
(A X, A Y)_{g}=(X, Y)_{g_{0}}
$$

for some (symmetric, positive definite) gauge transformation $A$ of the tangent bundle on $M_{\infty}$, where we identify the hyperbolic end $M_{\infty}$ with $H_{r}$. Setting $r=d_{g_{0}}\left(x_{0}, \cdot\right)$, then there exists an $\varepsilon>0$ such that

(i) $A$ is uniformly bounded from below and above;

(ii) $\left|\nabla^{g_{0}} A\right|+|A-I d|=O\left(e^{-(n+\varepsilon) r}\right)$ at infinity.

Theorem 1.1. If there exists a real function $H$ on strongly asymptotically hyperbolic spin manifold $(M, g)$ and $\varepsilon^{\prime}>0$,

$$
H=O\left(e^{-\left(n+\varepsilon^{\prime}\right) r}\right)
$$

Received January 13, 2000. 
at infinity such that

$$
R \geq-n(n-1)-n\left(H^{2}+2 H\right)+2 n|d H|,
$$

then $\left(M^{n}, g\right)$ is isometric to $\left(H^{n}, g_{0}\right)$. In particular, $H \equiv 0$.

This rigid theorem is true also for local and odd-dimensional complex hyperbolic spaces, as well as the multi-end case, this theorem of course holds for the assumption of strongly asymptotic hyperbolic via integral conditions on $A$ and $H$, for instance,

$$
e^{r}(A-I d) \in L^{1, p}, \quad e^{r} H \in L^{p}
$$

for $p \geq 1$.

The positive energy theorem for asymptotically flat spin manifolds whose scalar curvature satisfies

$$
R \geq-n\left(H^{2}+2 H\right)+2 n|d H|
$$

for some real function

$$
H=O\left(r^{-\left(1+\varepsilon^{\prime}\right)}\right)
$$

at infinity was considered in [9].

The positive energy theorem was first proved by Schoen and Yau via the geometric analysis method $[5,6,7]$. So it is interesting to use their method to prove these rigid theorems.

\section{Preliminaries}

Let $M$ be an (oriented) Riemannian spin manifold of dimension $n \geq 3$ with spinor bundle $S$. Let $g, g_{0}$ be Riemannian metrics on $M$. Let $A \in \operatorname{End}(T M)$ be the symmetric, positive definite 'gauge transformation' such that

$$
(A X, A Y)_{g}=(X, Y)_{g_{0}} .
$$

The gauge transformation $A$ induces a map from $S O\left(M, g_{0}\right)$ to $S O(M, g)$. Therefore it induces a map from $\operatorname{Spin}\left(n, g_{0}\right)$ to $\operatorname{Spin}(n, g)$ and hence a map from the spinor bundles $S\left(M, g_{0}\right)$ to $S(M, g)$ by

$$
A(X \cdot \phi)=(A X) \cdot(A \phi)
$$

Let $\nabla, \nabla^{g_{0}}$ be the Levi-Civita connections of $g, g_{0}$ respectively. We extend them to the spinor bundle $S$ and denote as $\nabla, \nabla^{g_{0}}$ also. To compare the two connections, we define a connection $\bar{\nabla}$ with respect to the metric $g$ by

$$
\bar{\nabla} X=A\left(\nabla^{g_{0}}\left(A^{-1} X\right)\right) \text {. }
$$

This connection has torsion

$$
\begin{aligned}
\bar{T}(X, Y) & =\bar{\nabla}_{X} Y-\bar{\nabla}_{Y} X-[X, Y] \\
& =-\left(\left(\nabla_{X}^{g_{0}} A\right) A^{-1} Y-\left(\nabla_{Y}^{g_{0}} A\right) A^{-1} X\right) .
\end{aligned}
$$


Since the connection $\nabla$ is torsionless with respect to the metric $g$, we obtain

$$
\begin{aligned}
2\left(\bar{\nabla}_{X} Y-\nabla_{X} Y, Z\right)_{g} & = \\
& (\bar{T}(X, Y), Z)_{g}-(\bar{T}(X, Z), Y)_{g}-(\bar{T}(Y, Z), X)_{g} .
\end{aligned}
$$

Now we compare $\nabla, \bar{\nabla}$ on spinor bundle $S$. Let $\left\{e_{i}\right\}$ be a local orthonormal frame for $g$, and $\left\{e^{i}\right\}$ be the local orthonormal coframe. Let $\left\{\sigma^{\alpha}\right\}$ be the local orthonormal frame of the spinor bundle. Denote by $\omega_{i j}, \bar{\omega}_{i j}$ the connection 1 -forms for $\nabla, \bar{\nabla}$ defined by

$$
\omega_{i j}=\left(\nabla e_{i}, e_{j}\right)_{g}, \quad \bar{\omega}_{i j}=\left(\bar{\nabla} e_{i}, e_{j}\right)_{g} .
$$

For spinor $\phi=\phi^{\alpha} \sigma_{\alpha}$, we have

$$
\begin{aligned}
& \nabla \phi=d \phi^{\alpha} \otimes \sigma_{\alpha}+\frac{1}{4} \sum_{i, j} \omega_{i j} \otimes e_{i} \cdot e_{j} \cdot \phi, \\
& \bar{\nabla} \phi=d \phi^{\alpha} \otimes \sigma_{\alpha}+\frac{1}{4} \sum_{i, j} \bar{\omega}_{i j} \otimes e_{i} \cdot e_{j} \cdot \phi .
\end{aligned}
$$

Therefore,

$$
\nabla \phi-\bar{\nabla} \phi=\frac{1}{4} \sum_{i, j}\left(\omega_{i j}-\bar{\omega}_{i j}\right) \otimes e_{i} \cdot e_{j} \cdot \phi .
$$

By (2.2) and (2.3), we obtain

$$
\left|\left(\omega_{i j}-\bar{\omega}_{i j}\right)\left(e_{k}\right)\right| \leq C\left|A^{-1}\right|\left|\nabla^{g_{0}} A\right|
$$

for some $C>0$. Therefore,

$$
\begin{aligned}
|\nabla X-\bar{\nabla} X| & \leq C\left|A^{-1}\right|\left|\nabla^{g_{0}} A\right||X|, \\
|\nabla \phi-\bar{\nabla} \phi| & \leq C\left|A^{-1}\right|\left|\nabla^{g_{0}} A\right||\phi|, \\
|D \phi-\bar{D} \phi| & \leq C\left|A^{-1}\right|\left|\nabla^{g_{0}} A\right||\phi|,
\end{aligned}
$$

where $X$ is a vector, $\phi$ is a spinor and $D, \bar{D}$ are the Dirac operators with respect to the connections $\nabla, \bar{\nabla}$.

\section{The Killing connections and Dirac operators}

The Killing connection on spinor bundle $S$ is defined by

$$
\hat{\nabla}_{X}=\nabla_{X}+\frac{\mathbf{i}}{2} X
$$


spinors vanish with respect to this connection are called imaginary Killing spinors. The curvature of connection $\hat{\nabla}$ is

$$
\begin{aligned}
\hat{R}(X, Y) \phi & =\left(\hat{\nabla}_{X} \hat{\nabla}_{Y}-\hat{\nabla}_{Y} \hat{\nabla}_{X}-\hat{\nabla}_{[X, Y]}\right) \phi \\
& =R(X, Y) \phi-\frac{1}{4}(X \cdot Y \cdot-Y \cdot X \cdot) \phi .
\end{aligned}
$$

Hence if a manifold $M$ has a local basis of imaginary Killing spinors, then $\hat{R}$ vanishes, so that $M$ has constant sectional curvature -1 and is locally isometric to hyperbolic space. If a manifold $M$ has an imaginary Killing spinor $\phi$, then

$$
0=\sum_{i} e_{i} \hat{R}\left(X, e_{i}\right) \phi=-\frac{1}{2}(\operatorname{Ric}(X)+(n-1) X) \cdot \phi,
$$

therefore $M$ has constant Ricci curvature $-(n-1)$.

The hyperbolic space $H^{n}$ has a full set of imaginary Killing spinors $\left\{\phi_{0}\right\}$, see $[1,3]$. Moreover, there is constant $C>0$ such that

$$
C^{-1} e^{-r} \leq\left|\phi_{0}\right|^{2} \leq C e^{r}
$$

as $r \rightarrow \infty$ by (3.1). This fact is very important in the proof of rigid theorems for strongly asymptotically hyperbolic manifolds.

Now we define a generalized Killing connection

$$
\tilde{\nabla}_{X}=\nabla_{X}+\frac{\mathbf{i}}{2}(1+H) X
$$

for some real function $H$, and a Dirac operator

$$
\tilde{D}=e_{i} \cdot \tilde{\nabla}_{i}=D-\frac{\mathbf{i}}{2} n(1+H)
$$

with respect to this generalized Killing connection. Since

$$
\begin{aligned}
d(\phi, \psi) * e^{i}= & \left(\nabla_{i} \phi, \psi\right) * 1+\left(\phi, \nabla_{i} \psi\right) * 1 \\
= & \left(\tilde{\nabla}_{i} \phi-\frac{\mathbf{i}}{2}(1+H) e_{i} \cdot \phi, \psi\right) * 1 \\
& +\left(\phi, \tilde{\nabla}_{i} \psi-\frac{\mathbf{i}}{2}(1+H) e_{i} \cdot \psi\right) * 1 \\
= & \left(\tilde{\nabla}_{i} \phi, \psi\right) * 1+\left(\phi, \tilde{\nabla}_{i} \psi-\mathbf{i}(1+H) e_{i} \cdot \psi\right) * 1, \\
d\left(e_{i} \cdot \phi, \psi\right) * e^{i}= & (D \phi, \psi) * 1-(\phi, D \psi) * 1 \\
= & \left(\tilde{D} \phi+\frac{\mathbf{i}}{2} n(1+H) \phi, \psi\right) * 1 \\
& -\left(\phi, \tilde{D} \psi+\frac{\mathbf{i}}{2} n(1+H) \psi\right) * 1 \\
= & (\tilde{D} \phi, \psi) * 1-(\phi, \tilde{D} \psi+\mathbf{i} n(1+H) \psi) * 1,
\end{aligned}
$$


we have

$$
\begin{aligned}
& \tilde{\nabla}_{i}^{*}=-\tilde{\nabla}_{i}+\mathbf{i}(1+H) e_{i} \cdot=-\nabla_{i}+\frac{\mathbf{i}}{2}(1+H) e_{i} \cdot, \\
& \tilde{D}^{*}=\tilde{D}+\mathbf{i} n(1+H)=D+\frac{\mathbf{i}}{2} n(1+H) .
\end{aligned}
$$

Note that

$$
\hat{\nabla}^{*} \hat{\nabla}=\hat{\nabla} \hat{\nabla}^{*}=\nabla^{*} \nabla+\frac{n}{4}
$$

Denote

$$
\hat{R}=R+n(n-1) .
$$

By (3.7), we have the following Weitzenböck formulas

$$
\begin{aligned}
\tilde{D}^{*} \tilde{D} & =\left(D+\frac{\mathbf{i}}{2} n(1+H)\right)\left(D-\frac{\mathbf{i}}{2} n(1+H)\right) \\
& =\nabla^{*} \nabla+\frac{1}{4}\left(R+n^{2}(1+H)^{2}\right)-\frac{\mathbf{i}}{2} n d H . \\
& =\hat{\nabla}^{*} \hat{\nabla}+\frac{1}{4}\left(\hat{R}+n^{2}\left(H^{2}+2 H\right)\right)-\frac{\mathbf{i}}{2} n d H \cdot, \\
\tilde{D}^{*} & =\left(D-\frac{\mathbf{i}}{2} n(1+H)\right)\left(D+\frac{\mathbf{i}}{2} n(1+H)\right) \\
& =\nabla^{*} \nabla+\frac{1}{4}\left(R+n^{2}(1+H)^{2}\right)+\frac{\mathbf{i}}{2} n d H . \\
& =\hat{\nabla}^{*} \hat{\nabla}+\frac{1}{4}\left(\hat{R}+n^{2}\left(H^{2}+2 H\right)\right)+\frac{\mathbf{i}}{2} n d H .
\end{aligned}
$$

Now we derive the integral forms of the Weitzenböck formulas. Since

$$
\begin{aligned}
d\left(e_{i} \cdot \phi, \tilde{D} \phi\right) * e^{i} & =\left((\tilde{D} \phi, \tilde{D} \phi)-\left(\phi, \tilde{D}^{*} \tilde{D} \phi\right)\right) * 1, \\
d\left(\phi, \hat{\nabla}_{i} \phi\right) * e^{i} & =\left((\hat{\nabla} \phi, \hat{\nabla} \phi)-\left(\phi, \hat{\nabla}^{*} \hat{\nabla} \phi\right)\right) * 1,
\end{aligned}
$$

then

$$
\begin{aligned}
& d\left(\phi, \nabla_{i} \phi+e_{i} \cdot D \phi-\frac{\mathbf{i}}{2}[n(1+H)-1] e_{i} \cdot \phi\right) * e^{i} \\
= & d\left(\phi, \hat{\nabla}_{i} \phi+e_{i} \cdot \tilde{D} \phi\right) * e^{i} \\
= & \left(|\hat{\nabla} \phi|^{2}-|\tilde{D} \phi|^{2}+\frac{1}{4}\left(\hat{R}+n^{2}\left(H^{2}+2 H\right)\right)|\phi|^{2}-\left(\phi, \frac{\mathbf{i}}{2} n d H \cdot \phi\right)\right) * 1 .
\end{aligned}
$$


Similarly,

$$
\begin{aligned}
& d\left(\nabla_{i} \psi+e_{i} \cdot D \psi+\frac{\mathbf{i}}{2}[n(1+H)-1] e_{i} \cdot \psi, \psi\right) * e^{i} \\
= & d\left(-\hat{\nabla}_{i}^{*} \psi+e_{i} \cdot \tilde{D}^{*} \psi, \psi\right) * e^{i} \\
= & \left(\left|\hat{\nabla}^{*} \psi\right|^{2}-\left|\tilde{D}^{*} \psi\right|^{2}+\frac{1}{4}\left(\hat{R}+n^{2}\left(H^{2}+2 H\right)\right)|\psi|^{2}+\left(\frac{\mathbf{i}}{2} n d H \cdot \psi, \psi\right)\right) * 1 .
\end{aligned}
$$

Let

$$
\hat{D}=e_{i} \cdot \hat{\nabla}_{i}=D-\frac{\mathbf{i}}{2} n
$$

and

$$
\tilde{R}_{ \pm}=\hat{R}+n^{2}\left(H^{2}+2 H\right) \pm 2 \mathbf{i} n d H \cdot .
$$

Note that (1.3) ensures that $\tilde{R}_{ \pm}$is nonnegative. Now we obtain

$$
\begin{aligned}
& \int_{\partial M}\left(\phi, \nabla_{i} \phi+e_{i} \cdot D \phi\right) * e^{i}-\int_{\partial M}\left(\phi, \frac{\mathbf{i}}{2}[n(1+H)-1] e_{i} \cdot \phi\right) * e^{i} \\
= & \int_{\partial M}\left(\phi, \hat{\nabla}_{i} \phi+e_{i} \cdot \hat{D} \phi\right) * e^{i}-\int_{\partial M}\left(\phi, \frac{\mathbf{i}}{2} n H e_{i} \cdot \phi\right) * e^{i} \\
(3.10)= & \int_{M}|\hat{\nabla} \phi|^{2}-|\tilde{D} \phi|^{2}+\frac{1}{4}\left(\phi, \tilde{R}_{-} \cdot \phi\right),
\end{aligned}
$$

and

$$
\begin{aligned}
& \int_{\partial M}\left(\nabla_{i} \psi+e_{i} \cdot D \psi, \psi\right) * e^{i}+\int_{\partial M}\left(\frac{\mathbf{i}}{2}[n(1+H)-1] e_{i} \cdot \psi, \psi\right) * e^{i} \\
= & \int_{\partial M}\left(-\hat{\nabla}_{i}^{*} \psi+e_{i} \cdot \hat{D}^{*} \psi, \psi\right) * e^{i}+\int_{\partial M}\left(\frac{\mathbf{i}}{2} n H e_{i} \cdot \psi, \psi\right) * e^{i} \\
(3.11)= & \int_{M}\left|\hat{\nabla}^{*} \psi\right|^{2}-\left|\tilde{D}^{*} \psi\right|^{2}+\frac{1}{4}\left(\tilde{R}_{+} \cdot \psi, \psi\right) .
\end{aligned}
$$

\section{Rigidity for strongly asymptotically hyperbolic manifolds}

Let $M$ be a complete spin manifold with $\hat{R}$ nonnegative and bounded. Let $C_{0}^{\infty}(S)$ be the space of smooth sections with compact support. Define an inner product on $S$ by

$$
(\phi, \psi)_{1}=\int_{M}(\nabla \phi, \nabla \psi)+\frac{n}{4}(\phi, \psi)
$$

and let $H^{1}(S)$ be the closure of $C_{0}^{\infty}(S)$ with respect to this inner product. Then $H^{1}(S)$ with the above inner product is a Hilbert space. Now define a bounded bilinear form $B$ on $C_{0}^{\infty}(S)$ by

$$
B(\phi, \psi)=\int_{M}(\tilde{D} \phi, \tilde{D} \psi) .
$$


By (3.10), we obtain

$$
\begin{aligned}
B(\phi, \phi) & =\int_{M}|\hat{\nabla} \phi|^{2}+\frac{1}{4}\left(\phi, \tilde{R}_{-} \cdot \phi\right) \\
& =\int_{M}|\nabla \phi|^{2}+\frac{n}{4}|\phi|^{2}+\frac{1}{4}\left(\phi, \tilde{R}_{-} \cdot \phi\right) .
\end{aligned}
$$

Since $\tilde{R}_{-}$is bounded also, we can extend $B$ to $H^{1}(S)$ as a coercive bilinear form if $\tilde{R}_{-}$is nonnegative.

We extend the imaginary Killing spinors $\left\{\phi_{0}\right\}$ on $M_{\infty}$ to the whole $M$. With respect to the metric $g$, these Killing spinors can be written as $\bar{\phi}_{0}=A \phi_{0}$. Now we show that $\hat{\nabla} \bar{\phi}_{0} \in L^{2}(S)$ : On $M_{\infty}$,

$$
\begin{aligned}
\hat{\nabla}_{X} \bar{\phi}_{0} & =\left(\nabla_{X}+\frac{\mathbf{i}}{2} X \cdot\right)\left(A \phi_{0}\right) \\
& =\left(\nabla_{X}-\bar{\nabla}_{X}\right)\left(A \phi_{0}\right)+\bar{\nabla}_{X}\left(A \phi_{0}\right)+\frac{\mathbf{i}}{2} X \cdot\left(A \phi_{0}\right) \\
& =\left(\nabla_{X}-\bar{\nabla}_{X}\right)\left(A \phi_{0}\right)+A\left(\nabla_{X}^{g_{0}} \phi_{0}\right)+\frac{\mathbf{i}}{2} X \cdot\left(A \phi_{0}\right) \\
& =\left(\nabla_{X}-\bar{\nabla}_{X}\right)\left(A \phi_{0}\right)-\frac{\mathbf{i}}{2}(A X)\left(A \phi_{0}\right)+\frac{\mathbf{i}}{2} X \cdot\left(A \phi_{0}\right) .
\end{aligned}
$$

Thus,

$$
\left|\hat{\nabla} \bar{\phi}_{0}\right| \leq C\left(\left|\nabla^{g_{0}} A\right|+|A-I d|\right)\left|\phi_{0}\right|
$$

by (2.6). Therefore,

$$
\begin{aligned}
\left|\hat{\nabla}_{X} \bar{\phi}_{0}\right| & \leq C\left(\left|\nabla^{g_{0}} A\right|+|A-I d|\right) e^{\frac{r}{2}}|X|, \\
\left|\hat{D} \bar{\phi}_{0}\right| & \leq C\left(\left|\nabla^{g_{0}} A\right|+|A-I d|\right) e^{\frac{r}{2}}
\end{aligned}
$$

as $r \rightarrow \infty$. Since the sphere $S_{r}$ has area of the order $e^{(n-1) r}$, we know that

$$
\hat{\nabla} \bar{\phi}_{0} \in L^{2}(S)
$$

and hence

$$
\hat{D} \bar{\phi}_{0} \in L^{2}(S)
$$

by the assumption of asymptotic hyperbolic metric. Therefore

$$
\tilde{D} \bar{\phi}_{0}=\hat{D} \bar{\phi}_{0}-\frac{\mathbf{i}}{2} n H \bar{\phi}_{0} \in L^{2}(S)
$$

by the assumption of behavior of $H$ at infinity. Note that $\bar{\phi}_{0}$ is not $L^{2}$ because $\left|\bar{\phi}_{0}\right|^{2} \geq C^{-1} e^{-r}$.

Lemma 4.1. There exists a unique spinor $\phi_{1}$ on $H^{1}(S)$ such that

$$
\tilde{D}\left(\phi_{1}+\bar{\phi}_{0}\right)=0
$$

if (1.3) holds. 
Proof. Since $B(\cdot, \cdot)$ is coercive on $H^{1}(S)$, and $\tilde{D} \bar{\phi}_{0} \in L^{2}(S), \hat{\nabla} \bar{\phi}_{0} \in L^{2}(S)$, the Lax-Milgram lemma shows that there exists a spinor $\phi_{1} \in H^{1}(S)$ such that

$$
\tilde{D}^{*} \tilde{D} \phi_{1}=-\tilde{D}^{*} \tilde{D} \phi_{0}
$$

weakly. Let $\phi=\phi_{1}+\bar{\phi}_{0}$ and $\psi=\tilde{D} \phi$. The elliptic regularity tells us that $\psi \in H^{1}(S)$, and

$$
\tilde{D}^{*} \psi=0
$$

in the classical sense. Then (3.11) implies

$$
\hat{\nabla}_{i}^{*} \psi=\nabla_{i} \psi-\frac{\mathbf{i}}{2}(1+H) e_{i} \cdot \psi=0 .
$$

Therefore,

$$
\left.\left.\left|\partial_{i}\right| \psi\right|^{2}|\leq(1+H)| \psi\right|^{2}
$$

Hence

$$
\left.\left|\partial_{i} \ln \right| \psi\right|^{2} \mid \leq 1+H
$$

on the complement of the zero set of $\psi$ on $M$. If there exists $x_{0} \in M$ such that $\left|\psi\left(x_{0}\right)\right| \neq 0$, then integrate it along a path from $x_{0} \in M$ gives

$$
|\psi(x)|^{2} \geq\left|\psi\left(x_{0}\right)\right|^{2} e^{(1+H)\left(\left|x_{0}\right|-|x|\right)} .
$$

Obviously, $\psi$ is not in $L^{2}(S)$ which gives the contradiction. Hence $\psi \equiv 0$, and the proof of lemma is complete.

Proof of Theorem 1.1. Let $\phi$ be the solution of $\tilde{D} \phi=0$ corresponding to the imaginary Killing spinor $\phi_{0}$ constructed in Lemma 4.1. Submit this $\phi$ into (3.10), we obtain

$$
\begin{aligned}
& \int_{S_{\infty}}\left(\phi, \hat{\nabla}_{i} \phi+e_{i} \cdot \hat{D} \phi\right) * e^{i}-\int_{S_{\infty}}\left(\phi, \frac{\mathbf{i}}{2} n H e_{i} \cdot \phi\right) * e^{i} \\
= & \int_{M}|\hat{\nabla} \phi|^{2}+\frac{1}{4}\left(\phi, \tilde{R}_{-} \cdot \phi\right) .
\end{aligned}
$$

Write $\phi=\phi_{1}+\bar{\phi}_{0}$ where $\phi_{1} \in H^{1}(S)$. Then

$$
\begin{aligned}
\left|\int_{S_{\infty}}\left(\phi, \hat{\nabla}_{i} \phi+e_{i} \cdot \hat{D} \phi\right) * e^{i}\right| & =\left|\int_{S_{\infty}}\left(\bar{\phi}_{0}, \hat{\nabla}_{i} \bar{\phi}_{0}+e_{i} \cdot \hat{D} \bar{\phi}_{0}\right) * e^{i}\right| \\
& \leq C \int_{S_{\infty}}\left(\left|\nabla^{g_{0}} A\right|+|A-I d|\right) e^{r}
\end{aligned}
$$

and

$$
\left|\int_{S_{\infty}}\left(\phi, \frac{\mathbf{i}}{2} n H e_{i} \cdot \phi\right) * e^{i}\right| \leq C \int_{S_{\infty}}|H| e^{r} .
$$

By the assumption on the asymptotic behaviors of $A$ and $H$ at infinity, we see that the left hand side of (4.3) vanishes, and hence

$$
\hat{\nabla} \phi=0 \text {. }
$$


Therefore

$$
0=\tilde{D} \phi=\hat{D} \phi-\frac{\mathbf{i}}{2} n H \phi=-\frac{\mathbf{i}}{2} n H \phi .
$$

This implies $H \equiv 0$. Thus it reduces to the case of Min-Oo's theorem [3] and the proof of the theorem is complete.

\section{References}

[1] L. Andersson and M. Dahl, Scalar curvature rigidity for asymptotically locally hyperbolic manifolds, Ann. Global Anal. Geom. 16 (1998), 1-27.

[2] M. Herzlich, Scalar curvature and rigidity of odd-dimensional complex hyperbolic spaces, Math. Ann. 312 (1998), 641-657.

[3] M. Min-Oo, Scalar curvature rigidity of asymptotically hyperbolic spin manifolds, Math. Ann. 285 (1989), 527-539.

[4] T. Parker and C. Taubes, On Witten's proof of the positive energy theorem, Comm. Math. Phys. 84 (1982), 223-238.

[5] R. Schoen, S.T. Yau, On the proof of the positive mass conjecture in general relativity, Comm. Math. Phys. 65 (1979), 45-76.

[6] R. Schoen and S.T. Yau, The energy and the linear momentum of spacetimes in general relativity, Comm. Math. Phys. 79 (1981), 47-51.

[7] R. Schoen and S.T. Yau, Proof of the positive mass theorem. II, Comm. Math. Phys. 79 (1981), 231-260.

[8] E. Witten, A new proof of the positive energy theorem, Comm. Math. Phys. 80 (1981), 381-402.

[9] X. Zhang, The positive mass theorem for modified energy condition, unpublished.

[10] X. Zhang, Angular momentum and positive mass theorem, Comm. Math. Phys. 206 (1999), 137-155.

Institute of Mathematics, Academy of Mathematics and System Sciences, Chinese Academy of Sciences, BeiJing 100080, P.R. China.

E-mail address: xzhang@math08.math.ac.cn 